\title{
Wound Dehiscence
}

National Cancer Institute

\section{Source}

National Cancer Institute. Wound Dehiscence. NCI Thesaurus. Code C50863.

Splitting of the sutured margins of a surgical wound. Risk factors include diabetes

mellitus, obesity, and advanced age. 